\title{
Synergistic effect of flow pattern evolution of dispersed and continuous phases in direct-contact heat transfer process
}

\author{
Document Version \\ Accepted author manuscript
}

Link to publication record in Manchester Research Explorer

Citation for published version (APA):

Xu, J., Liu, F., Xiao, Q., Huang, J., Fei, Y., Yang, Y., Zhai, Y., Pan, J., \& Wang, H. (2020). Synergistic effect of flow pattern evolution of dispersed and continuous phases in direct-contact heat transfer process. International Journal of Refrigeration, 112, 201-214.

\section{Published in:}

International Journal of Refrigeration

\section{Citing this paper}

Please note that where the full-text provided on Manchester Research Explorer is the Author Accepted Manuscript or Proof version this may differ from the final Published version. If citing, it is advised that you check and use the publisher's definitive version.

\section{General rights}

Copyright and moral rights for the publications made accessible in the Research Explorer are retained by the authors and/or other copyright owners and it is a condition of accessing publications that users recognise and abide by the legal requirements associated with these rights.

\section{Takedown policy}

If you believe that this document breaches copyright please refer to the University of Manchester's Takedown Procedures [http://man.ac.uk/04Y6Bo] or contact uml.scholarlycommunications@manchester.ac.uk providing relevant details, so we can investigate your claim.

\section{OPEN ACCESS}




\begin{abstract}
The marker-controlled multiple watershed segmentation are achieved to distinguish darker continuous phase in gas-liquid two-phase flow patterns efficiently. Each plot of the Betti numbers $\beta_{i}$ is curve-fitted using a fourparameter logistics model, for characterizing mixing effects. Similarity between adjacent pixels can be quantified by the distance. The $\beta_{1}$ of continuous phase decreases linearly at distance $\geq 2$, which can be used to determine the threshold for segmentation. Repeated tests with different pixels and meth-
\end{abstract}

*feiyukm@aliyun.com(Y.Fei);jianxin.pan@manchester.ac.uk(J.Pan);wanghua65@163.com(H.Wang)

${ }^{1}$ Equally contributing authors.

\title{
Synergistic effect of flow pattern evolution of dispersed and continuous phases in direct-contact heat transfer process
}

\author{
${ }^{a}$ State Key Laboratory of Complex Nonferrous Metal Resources Clean Utilization, \\ Kunming University of Science and Technology, Kunming, Yunnan, 650093, China \\ China \\ ${ }^{c}$ Faculty of Mechanical and Electrical Engineering, Yunnan Agriculture University, \\ Kunming, Yunnan, 650100, China \\ Handan, 056038, China \\ Kunming, 650221, China \\ Technology, Kunming, Yunnan, 650093, China \\ ${ }^{g}$ School of Mathematics, The University of Manchester, Manchester, M13 9PL, UK
}

Preprint submitted to International Journal of Refrigeration 
ods are conducted to ensure the repeatability and effectiveness of this model. More interestingly, we find that the rapid increase of $\beta_{1}$ of bubbles swarm coincides with the evolution of $\beta_{1}$ of continuous phase, and the median of difference of $\beta_{1}$ between the two phases in the visible window, as a novel metric of flow regime control is obtained and correlated with average volumetric heat transfer coefficient. This dynamic image analysis method, equipped with computational homology, provides the ability of evaluation of the spatial flow structure from a two-dimensional image and can be used to control the process.

Keywords: Distribution, Two phase flow, Heat transfer, Synergy, Image analysis

\section{1. Introduction}

2 Direct contact heat exchangers (DCHE) involve heat transfer between hot 3 and cold fluids of immiscible two phases in the absence of a separating wall, 4 where heat and mass are simultaneously transferred between the two phases 5 [1]. They are widely used in air conditioning[2], humidification[3], industrial 6 hot water heating[4], water cooling[5] and power engineering applications[6], 7 because of high thermal efficiency and low cost in comparison to a surface 8 type heat exchanger. Despite their high performance, it is still difficult to 9 obtain the correct heat transfer as a function of plant operating conditions

10 [7]. Garimella et al. investigated the flow distribution in micro-channel heat 11 exchanger manifolds and quantified the effects of inlet mass flux, inlet quality, 12 header flow regime and header pressure drop on distribution[8]. Mudawar et 
al. reported transverse profiles and stream-wise variations of void fraction, flow velocity, and wall temperature, and the computed results show reasonable agreement with experimentally captured interfacial behavior as well as heat transfer parameters[9]. Zhang et al. studied two-phase flow distribution in plate-fin heat exchangers, and found that the non-uniformity in the gas flow distribution decreased and that in the liquid flow non-uniformity increased with increasing inlet dryness fraction[10]. Two-phase flow maldistribution among the heat exchanger passages was more widespread compared to that of single-phase flow. More specifically, the liquid-phase distribution was more uneven compared to the gas-phase distribution. Liu et al. found that the phase distribution characteristics of two-phase flow in parallel channels highly depended on the inlet flow patterns and the orientations of header and channels[11]. Mei et al. investigated the phase distribution and the average void fraction in the cross section which were obtained from the local void fraction measurement by a conductivity probe[12]. Actually, the void fraction can quantify the two-phase distribution indirectly, but it can not describe the degree of dispersion or uniformity. Cascella et al.[7] reported the evolution of the velocity of the droplet as function their size in DCHE. The bubble size distributions together with the developed sparger model in direct-contact evaporators are used to estimate the three empirical parameters which are part of the population balance model. [13], and the results show an excellent agreement between calculated and experimental size distributions is verified. Masiukiewicz and Anweiler proposed a qualitative and quantitative description of the two-phase fluid flow structure based on grey 
level analysis and cross-correlation of signals[14], but usually this information is insufficient to accurately assess the flow conditions. Often only the number of bubbles is concerned, but the number change of continuous phase distribution is neglected. Additionally, both phase transformation and heat transfer increase the complexity of the problem.

Feature extraction and target segmentation are the main research contents of digital image processing technology. The support vector machine (SVM) was developed for pattern recognition, image classification, object detection $[15,16]$. With visualization study [17], the basic laws in the growth and development of the confined bubbles in ultra-shallow micro channel were clearly explained, and an interesting dividing criterion was proposed. Yang et al. [18] proposed a bubble image adaptive segmentation method to extract froth morphological feature (size and shape). Peyghambarzadeh et al.[19] found that detecting distinguishable bubbles was nearly impossible at high heat fluxes. Most actual industrial images are difficult to segment. The bubbles often touch each other with no void space between them. Moreover, the illumination on the bubble surface is uneven, and the edges among bubbles are normally weak, and the whole image is low contrast. Separating touching objects in an image is one of the more difficult image processing operations. The watershed segmentation is often applied to this problem. which is an image region segmentation method. In the process of segmentation, it will take the similarity with the neighboring pixels as an important reference, so as to connect pixels with similar spatial positions and similar gray values. The watershed transform finds catchment basins and watershed ridge 
lines in an image by treating it as a surface where light pixels are high and dark pixels are low. It is widely used with MRI[20, 21], CT[22], EUV[23], and SEM[24]. Recently, the marker-controlled watershed algorithms are used for data segmentation, in order to identify flow structures [25] and measure local and average partial wetting efficiencies [26]. However, using data segmentation watershed transform often results in over-segmentation because of the presence of too many local minima in the image. The simple marker controlled watershed segmentation solves the problem of over-segmentation [20], but cannot mark light bubbles and edges of heat transfer fluid properly (such as Fig.4). Besides, the intense agitation makes the liquid temperature highly uniform, eliminating points of super heating [1]. Many investigators have pointed out that the volumetric heat transfer coefficient, the holdup ratio and the heat transfer rate are affected strongly by the dispersed phase flow rate, while the continuous phase has no significant impact[27]. However, Mahood found that both the continuous phase and the dispersed phase flow rates have a significant positive impact on the average volumetric heat transfer coefficient[27]. The number of continuous phase dispersed in twophase flow patterns in visual window was coarsely characterised by Huang method $[28,29]$. Unlike most previous studies, which examined the motion law of dispersed phase in continuous phase and its influence on the transfer and reaction process, this work evaluated synergistic effect of flow pattern evolution of dispersed and continuous phases in direct-contact heat transfer process.

In addition, the zeroth Betti number $\beta_{0}$ and the first Betti number $\beta_{1}$ pro- 
vide the following geometric information: $\beta_{0}$ equals the number of connected components that make up the space, and the first Betti number $\beta_{1}$ provides a measure of the number of tunnels in the structure. In 2-dimensional cases, $\beta_{0}$ is the number of connected components, which simply represents the number of pieces in the patterns. The number of these holes which are completely enclosed by cubes/pixels is measured by $\beta_{1}$. Limited by available methodologies, the synergistic effect of gas-liquid two-phase flow patterns and heat exchanger efficiency has not been quantified directly. Fortunately, the number of objects that result from segmentation depends on the number of local minimum that exists in the image [20]. In this work, continuous phase image segmentation conducted with marker-controlled multiple watershed segmentation, combined with algebraic topology has been introduced, which provides effective segmentation results that can mark light bubbles and continuous phase distribution efficiently. The scientific contribution of this paper is to discover the mechanism of the synergistic effect of dispersed and continuous phases distribution on the average volume heat transfer coefficient in boiling heat transfer process from the perspective of topology.

\section{Materials and methods}

\subsection{Materials}

The mixture of heat transfer fluid and organic working fluid is examined in a direct-contact heat exchanger. HTF(THERMINNOL(B)66) is used as the continuous phase in the DCHE. The refrigerant R-245fa (1,1,1,3,3 pentafluoropropane) is used as the dispersed phase, and its boiling point is $15.3^{\circ} \mathrm{C}$ in 
their standard state.

\subsection{Experimental setup}

The schematic of the visualized experimental system and the evolution of gas/liquid two phase flow are shown as Fig.1. Visualization study on flow boiling in direct-contact evaporator has been conducted under various operation conditions. A total of nine valid cases using an orthogonal array, $L_{9}$ orthogonal experimental design (OED) are selected to analyze topological characteristics and synchronization of gas-liquid two-phase flow patterns in the direct-contact evaporator in Tab.2. Four design parameters including the height of the HTF, the initial heat transfer temperature difference, and the flow rate of refrigerant and HTF are selected to investigate the evolution of gas-liquid two phase flow patterns in Tab.1. The test device with two circulation loops are shown in Fig.1 (one is HTF loop, the other is dispersed phase loop). These bubbles images are captured by a high-speed camera (the brand is PRAKTICA from Germany), and analyzed using digital image processing technology. As experimental errors inevitably exist, In Fig.11 (right) repeated tests are conducted to ensure the repeatability and effectiveness of the experimental phenomena.

\subsection{Betti numbers}

Algebraic topology provides quantitative information on complex structures [30]. Topologically invariant Betti numbers are used to examine the structures of flow patterns of interest in any dimension, which are coarser measures of this information. Quantifying the topology of these gas-liquid 
flow patterns is our goal as it will provide us with an additional metric for the structure of evolution. In the case of a $2 \mathrm{D}$ structure, there are two Betti numbers. $\beta_{0}$ is defined as the number of connected components, $\beta_{1}$ is the number of tunnels within a structure. A free software called Computational Homology Project (CHomP) is used to calculate the Betti numbers in digital datasets[31]. To use this program, one generates binary images of boiling bubbles and CHomP calculates Betti numbers. It is noted that CHomP provides estimation for Betti numbers. When comparing the $\beta_{0}$ or $\beta_{1}$ value, which should reflect the number of bubbles or HTF contained in the gas-liquid mixture. In this work, to obtain these binary images for estimating Betti numbers, the image processing methods such as watershed transformation and thresholding method are introduced.

The Betti numbers can be used to quantitatively distinguish between micro-structure morphologies generated by different models [30]. Fig.2 shows the evolution of Betti numbers $\left(\beta_{0}, \beta_{1}\right)$ for quantifying gas-liquid two-phase flow patterns. The red curves of $\beta_{1}$ is representative of the number of connected components, leading to a useful parameter for quantifying the number of bubbles. The blue curves of $\beta_{0}$ is the number of holes or tunnels in 2D bubble image, which is coarser measures of HTF phase. It is found that the time profile of the variation of the Betti number $\beta_{1}$ can give the description of the mixing time (inflection point) and the degree of homogeneity (the value of Betti number) in the evaporator. However, the evolution of the Betti number $\beta_{0}$ reflects random unordered fluctuations. This paper aims to give a good solution for characterizing the number of continuous phase distribution 
within gas-liquid two-phase flow patterns.

\subsection{Feature extraction methods}

In previous studies, the extraction of digital features of bubble group images in direct contact heat exchanger was implemented by Huang method[28], which included top-hat transform, binarization, and open operation. The top-hat transform is used to suppress the background of the original image and to eliminate noise and enhance the image. The binarization operation could be used for the computation of the Betti numbers. In a binary image, the white area refers to the bubble swarm, while the remaining black area indicates the continuous phase. The purpose of open operation is to remove tiny points, isolate points at the finer locations, and smoothen the boundaries of larger points without significantly changing the size of the image. It is used to eliminate small holes that represent single bubbles or small bubble groups in the binarization images.

Support vector machines has been proposed as a very effective method for pattern recognition(Cortes Vapnik, 1995). Intuitively given a set of points which belong to either one of the two classes, a SVM can find a hyperplane having the largest possible fraction of points of the same class on the same plane. The optimal separating hyperplane can minimize the risk of misclassifying examples of the test set.

The watershed transform is one of the most useful methods in field of image segmentation[32], which finds "catchment basins" or "watershed ridge lines" in an digital image by considering it as a surface where light pixels 
represent high elevations and dark pixels represent low elevations.

In this study, the continuous phase image segmentation conducted with marker-controlled multiple watershed transform is used to obtain accurate edge detection and the number of HTF phase. An efficient implementation of this method is as following. Firstly, the gradient image is obtained by applying the Sobel filter on the bubbles swarm image, and then the first watershed transform is conducted. the regional minima of gradient image is computed, which are connected components of pixels with a constant intensity value. Moreover, the extended-minima is computed to obtain the regional minima of H-minima transform in Matlab software. Secondly, the second watershed segmentation combined with Euclidean distance transform is conducted, and then the mimima imposition transform is applied to modify intensity image using morphological reconstruction so it only has regional minima at one location. Finally, the third watershed segmentation is then carried out. In addition, OSTU thresholding[20], filling operation and suppressing border structures are used to obtain the desired results (As is shown in Fig.4).

\section{Results and discussion}

\subsection{Performance analysis of watershed transform combined with homological} characterization

The watershed segmentation simulates the rain over the surface associated with the gray image[32] (As is seen in Fig.4). The drops will flow along 
the path of steepest descent until reaching the point of minimum, which is labeled as belonging to the reception basin associated with this local minimum. However, limitations of watershed segmentation are over segmentation and its sensitivity to noise. Fig.3 shows over segmentation produced by general segmentation method (left) and gradients segmentation method (right). In order to solve the problem of over-segmentation and feature extraction, the marker-controlled multiple watershed segmentation combined with thresholding and filling operations are illustrated in Fig.4.

In Fig.5 the $\beta_{1}$ obtained by Huang method represents the number of bubbles swarm, and the $\beta_{0}$ of binary image is the number of holes within gasliquid mixture, which may measure coarsely the number of the continuous phase distribution. Based on marked matrix by the watershed transform, an accurate edge detection defined by a set of connected pixels is obtained. The continuous phase (HTF) feature extraction combined with thresholding and filling operations is used to compute the Betti numbers of gas-liquid two-phase flow pattern. The results are compared with Huang method and SVM method. The method presented can obtain an accurate HTF detection in gas-liquid two phase flow pattern, in contrast with SVM method. In Fig.6 the $\beta_{0}$ of binary image is 4 , and the $\beta_{1}$ of binary image is 494 . However, by using the naked eye, at least eight objects labeled by the red ellipses are unrecognizable in this image. In order to verify the validity of the watershed transform method, a random bubble diagram is used to estimate the number of continuous phases, and then the influence of threshold on flow regimes identification is discussed. 
The accuracy and precision of the watershed transform method in different thresholds are shown in Fig.7. The distance $\mathrm{h}$ is a non-negative scalar. The over-segmentation occurs in the area near the minimum value with distance $=0$ and 1, which is marked by red rectangle in Fig.7 (top). In Fig.7 (top-right), the area marked by the red ellipse is incapable of identification with distance=1. In Fig.7 (bottom-right), the area marked by the blue ellipse indicates that the droplet can not be identified with distance=3. Fig.7 (bottom-left) illustrates a relatively precise segmentation with distance $=2$. However, the influence of distance near the minimum value on the first Betti numbers of HTF phase should be further discussed.

Merging the catchment basins is the best solution to solve over-segmentation based on a continuous iteration over the watershed transform[32]. Generally, each iteration calculates the neighbor catchment basins that might be joined with a lower cost and merges them by various criteria, such as an established threshold. In this paper, the minimum transformation of image is calculated to extend the local minimum area by a threshold, so that more pixels that are similar to the minimum area can be merged into the smallest area. However, the choice of threshold includes usually a subjective element. In order to overcome the limitation of this subjective method, a characteristic curve combined with linear fitting can be used to determine the threshold for extending the local minimum area (as is seen in Fig.8).

Fig.8 illustrates the relationship between threshold and Betti numbers of HTF phase, which shows a highly linear correlation when the distance is equal to 2. According to the value $\beta_{1}=507$ of HTF phases estimated 
by the SVM method, the numerical interval of HTF phases estimated with the increasing distance can be coarsely determined between 369 and 1131 in Fig.8 (left). The linear profile of Betti numbers is becoming more obvious with increasing thresholds (distance), and it is also verified experimentally that the number of HTF phases can be quantified precisely by this method (as is shown in Fig.7). Almost all the targets that can be seen by the naked eye are marked at the case of distance $=2$. Finally, experimental results of $L_{1}-L_{9}$ show these characteristic curves combined with good linear fitting can be used to determine the threshold for segmentation of the HTF phases.

\subsection{Evolution and synchronization of gas-liquid two-phase flow structures}

In the previous work, the bubbles swarm flow structures have been quantified using computational homology [28]. The whole life-cycle of boiling bubbles is involved in generation, split, merger and annihilation. An empirical growth curve is a scatter plot of some measure of the size of an object or individual against time $x$. It is generally assumed that, apart from some form of random fluctuation, the underlying growth follows a smooth curve. In addition, the analysis of growth data is very important in many fields of study such as biology, chemistry, sociology, and management [33]. There have been two main methods to analyze growth-curve data, namely statistical and biological. The statistical method is purely empirical and involves fitting polynomial curves to the data using multivariate models. However, their parameters have no physical interpretation, and they do not model subject-matter knowledge of the growth process. It is usually quite inap- 
propriate for growth studies [33]. Bubbles growth is of interest in boiling heat transfer process, as is the growth of continuous phase. In this paper we are interested in knowing how fast they grow, the effect of gas/liquid two phase mixing upon such growth and how these factors respond to heat transfer performance. Various researchers have used logistic model to predict the population growth [34] Inspired and motivated by growth model, the four-parameter logistic model is introduced to fit the evolution curves[33]. It is better thought of as empirical model which has known nonlinear behavior built into them and which has physically meaningful parameters. This model is characterized by its classic $\mathrm{S}$ or sigmoidal shape that fits the bottom and top plateaus of the curve. Phases shown in the sigmoid curve can also be explained by rates of generation, disappearance, merge and rupture. As the name implies, it has four parameters that need to be estimated in order to fit the curve. The equation for the model is [33]:

$$
y=d+\frac{a-d}{1+\left(\frac{x}{c}\right)^{b}}
$$

where $\mathrm{a}$ is the minimum value, $\mathrm{d}$ is the maximum value, $\mathrm{c}$ presents the point of inflection (i.e. the point on the $\mathrm{S}$ shaped curve halfway between a and d), b is the Hills slope of the curve. This model, known as the four-parameter logistic model, is mentioned in [33].

Fig.9 illustrates the fitting curves of Betti numbers evolution of the bubbles swarm. It is found that the evolution of these bubbles swarm typically follows four key stages: a lag phase, an exponential growth phase, a transitional phase and a plateau phase. The profile of mixing time is obtained for 
characterizing mixing effects. This methodology has been tested with 4500 images, taken from 9 videos recorded by the high-speed shutter video camera. Clearly, the different mixing performances are illustrated in Fig.9. An interesting tendency is found in the better cases of $L_{6}$, in which the larger first Betti numbers average and shorter mixing time $\tau$ correspond to a higher volumetric heat transfer coefficient. Combined with mixing index in [28], a new characteristic curve can be applied to quantify the heat transfer performance and mixing effects of bubble swarm patterns. The HTF phase features of gas-liquid two-phase flow patterns in the DCHE can be extracted and characterized by watershed transform method. In order to further quantify the topological properties of the HTF phase, the effects of threshold selection (distance) on its growth data are investigated by four-parameter logistics model. Each plot obtained from the bubbles evolution sample is curve-fitted using a four-parameter logistics model. In Fig.10 (top), the over-sampling occurs at distance $=0$ and distance $=1$. Fig. 10 shows that the evolution profile of mixing time is becoming more obvious with increasing distance $\geq 2$. More interestingly, we find that the population of $\beta_{1}$ of HTF and bubbles show a similar trend. Fig.11 (left) illustrates the effect of distance of pixels on sigmoidal shape curves fitted by four-parameter logistics model. We find that the size of $\beta_{1}$ of HTF phase decreases linearly from average $=55$ to average $=12$, and those curves show a similar stabilities. In Fig.11 (right), a repeated experiment at $L_{1}$ is used to test the validity of the model with different pixels of $291 \times 251$ (blue) and $1280 \times 720($ red). We also find a similar stabilities with standard deviation $\delta=62$ (red) and $\delta=61.4$ (blue). This 
means that our method is not limited to the type and pixels of the camera, and can be widely used.

In Fig.12, the growth curves of $\beta_{1}$ in $L_{9}$ fitted by four-parameter logistics model are compared by using the Huang method, SVM method, and watershed transform method. Fig.12 (left) shows that the size growth of bubbles swarm follows a smooth curve apart from some form of random fluctuation, and the evolution of $\beta_{0}$ of HTF cannot be characterized by the sigmoidal shape. In Fig.12 (right) we also find a similar trend by the SVM and watershed transform, and a significant difference in the size of continuous phase, which further validates the effectiveness of the watershed transformation method. A new characteristic curve for quantifying the evolution of continuous phase is obtained.

Both support vector machine and watershed transformation methods can be used to recognize continuous phase flow pattern. 500 frames of flow patterns in gas-liquid mixing process can be obtained by image processing technology, and one of them is used to illustrate the error between the two methods in Fig.6. The calculation results show that watershed transformation method has better advantages in identifying continuous phase features. The existence of noise in the experiment makes it difficult to accurately obtain the number of continuous phase dispersion in mixture images. However, there must be some errors between the observed values and the real values, which is hard to be quantified directly. In view of this, this paper compares the difference between support vector machine and watershed transformation method is constructed to describe the relative accuracy of watershed algorithm. 
Further more, Fig.13 shows evolution plots of continuous phase topological invariants by Huang method, SVM and watershed transform. Also, 500 images in each experiment are used to estimate the Betti numbers of continuous phase. The relative errors between support vector machine and watershed transformation method are shown in yellow lines. Compared with other froth image segmentation method, the proposed method achieves much high accuracy.

Next, synergistic effect of topological evolution of continuous and dispersed phase flow patterns on heat transfer performance in a direct contact heat exchanger is investigated and discussed.

In Fig.14, the black and green curves fitted by four-parameter logistic model show obvious difference, suggesting that coupling degree of gas-liquid two phase (bubbles swarm and HTF phase) topological invariants $\left(\beta_{1}\right)$ is correlated with heat exchanger efficiency. The change in the Betti number of bubbles phase obtained using the Huang method (blue points) is consistent with these of HTF phase extracted by the watershed transform (red points). Interestingly, the $\beta_{1}$ values for gas-liquid two phase flow structures still follow the " $\mathrm{S}$ " curve under experimental noise $\left(L_{2}, L_{3}\right)$, indicating that the experimental lighting does not affect the stabilities.

To quantify the topological structures of gas-liquid two phase flow in DCHE, we define an index median $\left(\Delta \beta_{1}\right)$ for characterizing the difference between the topological invariant $\beta_{1}$ of gas-liquid two phase flow patterns (as is shown in Tab.3).

The volumetric heat transfer coefficient of a direct contact heat exchanger 
has been investigated experimentally [27, 35, 36, 28]. To evaluate the effect of the topological structures of gas-liquid two phase flow on heat exchanger efficiency, the average volume heat transfer coefficient $\overline{h_{v}}$ and its growth rate (slope of $h_{v}$ ) are calculated in Tab.3. The volumetric heat transfer coefficient, $h_{v}$ is as follows.

$$
\begin{aligned}
& h_{v}=\frac{m_{g}\left(h_{d o}-h_{d i}\right)}{V \Delta T} \\
& \Delta T=\frac{\left(T_{c i}-T_{d o}\right)-\left(T_{c o}-T_{d i}\right)}{\ln \frac{T_{c i}-T_{d o}}{T_{c o}-T_{d i}}}
\end{aligned}
$$

337

where $\mathrm{m}$ is mass flow-rate of the dispersed phase steam, and $\mathrm{h}$ is the enthalpy of the dispersed phase, $\mathrm{T}$ is temperature. The $\Delta T$ in Equ.(2) is the logarithmic mean temperature difference.

Fig.15 (A) shows the median $\Delta \beta_{1}$ of gas-liquid two-phase flow structures vs slope of $h_{v}$ with correlation coefficient $\rho=-0.85$, suggesting that the heat exchanger efficiency is correlated with topological features of gas-liquid two phase flow patterns. In Fig.15 (B), the slope of $h_{v}$ (red) and average of $h_{v}$ (blue) show similar trends with $\rho=0.94$. As expected, in Fig.15 (C), the median $\Delta \beta_{1}$ of gas-liquid two-phase flow structures and average of $h_{v}$ show a highly negative correlation within experimental error with $\rho=-0.82$. The results shows that the average volumetric heat transfer coefficient is reduced as the $\Delta \beta_{1}$ of gas-liquid two-phase flow structures increased. Also, both the continuous phase and the dispersed phase topological invariants have a significant impact on the average volumetric heat transfer coefficient. Our results provide evidence for topological couping of gas-liquid two phase flow patterns and heat exchanger performance. 


\section{Conclusions}

In this paper, the heat transfer to dispersed droplets in an immiscible continuous phase is studied for the R245fa-HTF system. The marker-controlled multiple watershed segmentation are used for feature extraction of the gasliquid two-phase flow patterns. Topological invariants are applied to estimate the accurate numbers of pieces obtained by Huang method, SVM and watershed transform method. Experimental results indicate the watershed transform is suitable and accurate for darker targets in the gas-liquid twophase flow patterns. A four parameter logistic function is used to describe the evolution of the bubbles swarm and HTF phase at different conditions, leading a useful characteristic curve to characterize mixing performance. Effects of similarity between adjacent pixels (distance) on the Betti numbers of pieces are discussed. Experimental results show that the $\beta_{1}$ of continuous phase decreases linearly at distance $\geq 2$, which can be used to determine the threshold for segmentation. The evolution profile of mixing time is becoming more obvious with the increasing distance. As experimental errors inevitably exist, repeated tests with different pixels and methods are performed to ensure the repeatability and effectiveness of this model. More interestingly, we find that $\beta_{1}$ time series of bubbles swarm and continuous phase show a synchronous evolution. Coupling degree of Betti numbers from the two phases can be quantified by the median of difference of $\beta_{1}$. Experimental results show the median of difference of $\beta_{1}$ between the two phases is correlated with the growth rate of heat transfer coefficient (slope of $h_{v}$ ). The varia- 
tion of average of $h_{v}$ coincides with the evolution of slope of $h_{v}$, suggesting that coupling degree of gas-liquid two phase (bubbles swarm and continuous phase) topological invariants $\left(\beta_{1}\right)$ is correlated with heat exchanger efficiency.

It has been proposed that new enhanced heat transfer mechanism in DCHE can be illustrated via topological coupling between gas-liquid twophase flow patterns and heat exchanger efficiency from a flow pattern control perspective. We anticipate that these topological invariants will be instrumental in studying the interplay between the topology and thermal efficiency of enhanced mixing flow pattern. In summary, the topological coupling between gas-liquid two-phase flow patterns and heat exchanger efficiency provides evaluation of the spatial flow structure from a two-dimensional image and can be used to control the process.

\section{Acknowledgements}

We wish to thank the referees for their numerous detailed questions and constructive criticism, which greatly improved the presentation. This work is partially supported by the National Natural Science Foundation of China (Grant Nos.51666006, 51706195, 11971421), Joint Funds of the National Natural Science Foundation of China (Project No.U1602272), and Natural Science Foundation of Yunnan province (Project No.2017FB093).

[1] P. L. d. C. Lage, F. B. Campos, Advances in direct contact evaporator design, Chemical Engineering and Technology 27 (2004) 91-95.

[2] X. Y. Li, Q. Q. Zhao, D. Q. Qu, Investigation on the dynamic character- 
istics of a direct contact thermal energy storage charging process for use in conventional air-conditioning systems, Applied Thermal Engineering 91 (2015) 172-180.

[3] T. Ke, X. Huang, X. Ling, Numerical and experimental analysis on air/water direct contact heat and mass transfer in the humidifier, Applied Thermal Engineering 156 (2019) 310-323.

[4] S. R. Yang, J. Seo, Y. A. Hassan, Thermal hydraulic characteristics of unstable bubbling of direct contact condensation of steam in subcooled water, International Journal of Heat and Mass Transfer 138 (2019) 580596.

[5] Z. Zeng, A. Sadeghpour, Y. S. Ju, Thermohydraulic characteristics of a multi-string direct-contact heat exchanger, International Journal of Heat and Mass Transfer 126 (2018) 536-544.

[6] K. Zhu, J. Xia, X. Xie, Y. Jiang, Total heat recovery of gas boiler by absorption heat pump and direct-contact heat exchanger, Applied Thermal Engineering 71 (2014) 213-218.

[7] F. Cascella, A. Teyssedou, Modeling a Direct Contact Heat Exchanger used in a supercritical water loop, Applied Thermal Engineering 79 (2015) 132-139.

[8] A. J. Mahvi, S. Garimella, Two-phase flow distribution of saturated refrigerants in microchannel heat exchanger headers, International Journal of Refrigeration 104 (2019) 84-94. 
[9] J. Lee, L. E. O’Neill, S. Lee, I. Mudawar, Experimental and computational investigation on two-phase flow and heat transfer of highly subcooled flow boiling in vertical upflow, International Journal of Heat and Mass Transfer 136 (2019) 1199-1216.

[10] Z. Zhang, S. Mehendale, J. J. Tian, Y. Z. Li, Experimental investigation of two-phase flow distribution in plate-fin heat exchangers, Chemical Engineering Research and Design 120 (2017) 34-46.

[11] Y. Liu, W. Sun, W. Wu, S. Wang, Gas-liquid two-phase flow distribution in parallel micro-channels with different header and channels orientations, International Journal of Heat and Mass Transfer 112 (2017) $767-778$.

[12] Y. Mei, S. Gong, H. Gu, W. Ma, A study on steam-water two phase flow distribution in a rectangular channel with different channel orientations, Experimental Thermal and Fluid Science 99 (2018) 219-232.

[13] C. P. Ribeiro, P. L. C. Lage, Population balance modeling of bubble size distributions in a direct-contact evaporator using a sparger model, Chemical Engineering Science 59 (2004) 2363-2377.

[14] M. Masiukiewicz, S. Anweiler, Two-phase flow structure assessment based on dynamic image analysis, Flow Measurement and Instrumentation 65 (2019) 195-202.

[15] V. N. Vapnik, Statistical Learning Theory, Adaptive and learning Systems for Signal Processing, Communications and Control 2 (1998) 1-740. 
[16] W. Zhang, F. Y. Shih, N. Jin, Y. Liu, Recognition of gas liquid twophase flow patterns based on improved local binary pattern operator, International Journal of Multiphase Flow 36 (2010) 793-797.

[17] S. Hong, Y. Tang, Y. Lai, S. Wang, L. Zhang, Visualization research on confined bubble growth feature and heat transfer characteristic in ultra-shallow micro channel, International Journal of Heat and Mass Transfer 103 (2016) 847-854.

[18] K.-j. ZHOU, Q. Zhao, J. Xiang, J. Shi, L. Wang, S. Hu, S. Su, Clustering-driven watershed adaptive segmentation of bubble image, J. Cent. South Univ. Technol. 17 (2010) 1049-1057.

[19] S. M. Peyghambarzadeh, M. M. Sarafraz, N. Vaeli, E. Ameri, A. Vatani, M. Jamialahmadi, Forced convective and subcooled flow boiling heat transfer to pure water and n-heptane in an annular heat exchanger, Annals of Nuclear Energy 53 (2013) 401-410.

[20] G.-r. Kwon, D. Basukala, S.-w. Lee, K. H. Lee, M. Kang, Brain Image Segmentation Using a Combination of Expectation-Maximization Algorithm and Watershed Transform, International Journal of Imaging Systems and Technology 26 (2016) 225-232.

[21] J. C. Richard Beare, Brain extraction using the watershed transform from markers, Frontiers in Neuroinformatics 7 (2013) 1-15.

[22] W.-y. Hsu, Improved watershed transform for tumor segmentation : 
Application to mammogram image compression, Expert Systems With Applications 39 (2012) 3950-3955.

[23] M. Ciecholewski, Automated coronal hole segmentation from Solar EUV Images using, Journal of Visual Communication and Image Representation 33 (2015) 203-218.

[24] Y. Zhu, T. Zuo, L. Jiang, Z. Cai, C. Yan, X. Liu, X. Malcolm, Q. Wu, Pore structure analysis on activated carbon fibers By cluster and watershed transform method, Applied Surface Science 256 (2009) 587-592.

[25] G. Farideh, A. Ronnie, A. Bengt, Identication and Characterization of Three-Dimensional Turbulent Flow Structures, AIChE Journal 62 (2016) 1265-1277.

[26] L. Baussaron, C. Julcour-Lebigue, A.-M. Wilhelm, H. Delmas, Wetting Topology in Trickle Bed Reactors, AIChE Journal 53 (2007) 1850-1860.

[27] A. S. Baqir, H. B. Mahood, A. N. Campbell, A. J. Griffiths, Measuring the average volumetric heat transfer coefficient of a liquid-liquid-vapour direct contact heat exchanger, Applied Thermal Engineering 103 (2016) $47-55$.

[28] J. Huang, J. Xu, X. Sang, H. Wang, H. Wang, Quantifying the synergy of bubble swarm patterns and heat transfer performance using computational homology, International Journal of Heat and Mass Transfer 75 (2014) 497-503. 
[29] J. Xu, H. Wang, H. Fang, Multiphase mixing quantification by computational homology and imaging analysis, Applied Mathematical Modelling 35 (2011) 2160-2171.

[30] M. Gameiro, K. Mischaikow, T. Wanner, Evolution of pattern complexity in the Cahn-Hilliard theory of phase separation, Acta Materialia 53 (2005) 693-704.

[31] Tomasz Kaczynski, Konstantin Mischaikow, Marian Mrozek, Computational Homology, volume 59, 2010.

[32] V. Osma-Ruiz, J. I. Godino-Llorente, N. Sáenz-Lechón, R. Fraile, Segmentation of the glottal space from laryngeal images using the watershed transform, Computerized Medical Imaging and Graphics 32 (2008) $193-201$.

[33] G. A. F. SEBER, Nonlinear Regression Mathematical Modelling, 23, John Wiley \& Sons, Inc., Auckland, 2007.

[34] D. Mohapatra, J. M. Frias, F. A. R. Oliveira, Z. M. Bira, J. Kerry, Development and validation of a model to predict enzymatic activity during storage of cultivated mushrooms (Agaricus bisporus spp.), Journal of Food Engineering 86 (2008) 39-48.

[35] A. S. Baqir, H. B. Mahood, M. S. Hameed, A. N. Campbell, Heat transfer measurement in a three-phase spray column direct contact heat exchanger for utilisation in energy recovery from low-grade sources, Energy Conversion and Management 126 (2016) 342-351. 


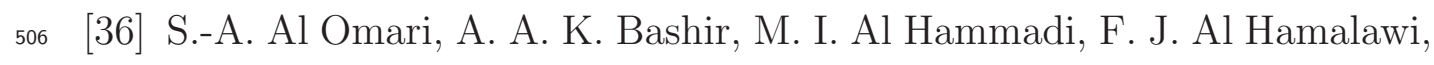
${ }_{507}$ E. Elnajjar, A. M. Ghazal, Experimental assessment of a direct-contact 508 heat exchanger bubbling hot water in a cooler liquid gallium bath, Inter509 national Communications in Heat and Mass Transfer 86 (2017) 71-80. 


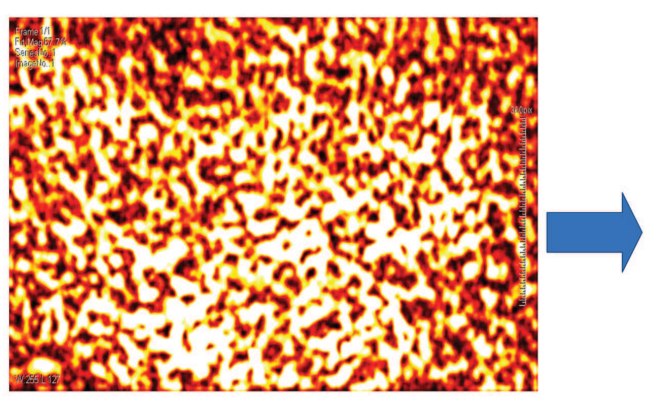

Image of gas-liquid mixture

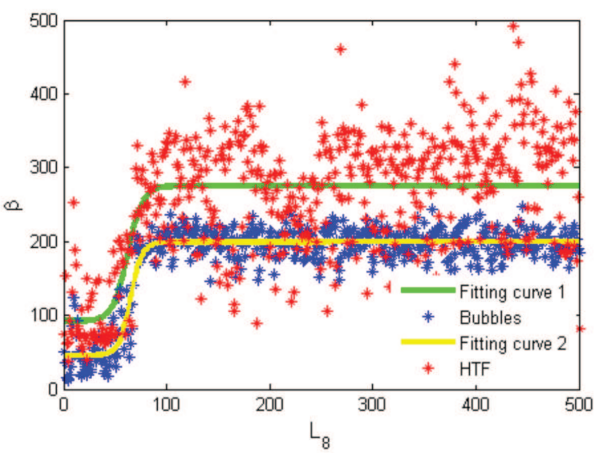

Betti numbers evolution of gas-liquid two phase

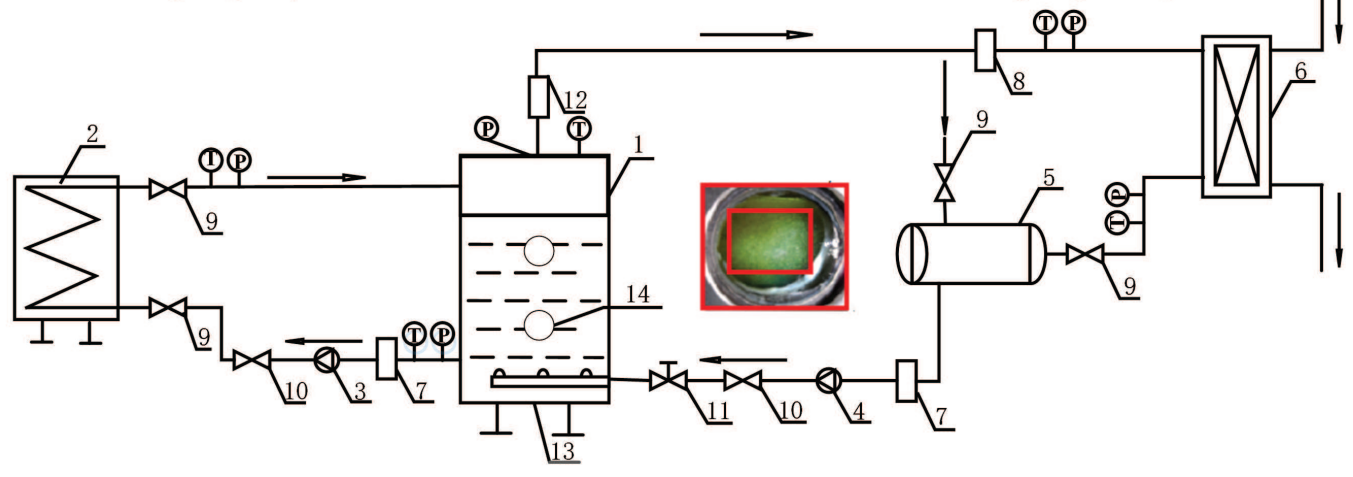

Figure 1: Sketch of the experimental set-up.(1-Direct contact heat exchanger, 2-electric heater, 3-heat transfer fluid pump, 4-centrifugal pump, 5-fluid reservoir, 6-plate condenser, 7-mass flowmeter, 8-flow meter, 9-globe valve, 10-check valve, 11-regulating valve, 12-Gasliquid separator, 13-spray head, 14-visual window) 

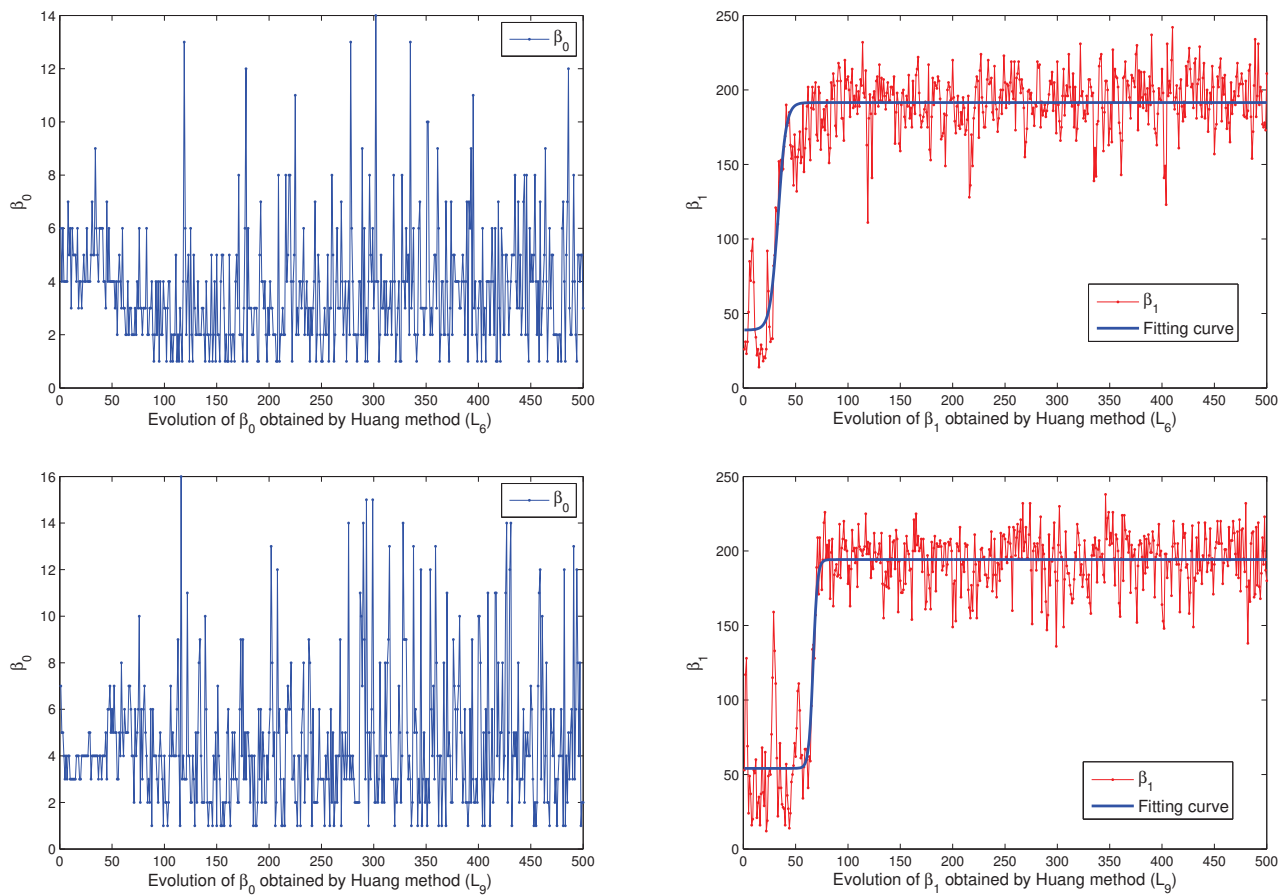

Figure 2: Evolutions of Betti numbers $\left(\beta_{0}, \beta_{1}\right)$ for quantifying gas-liquid two-phase flow patterns

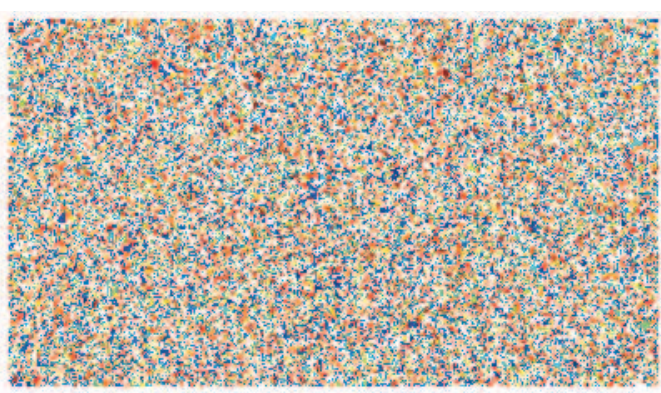

General segmentation method

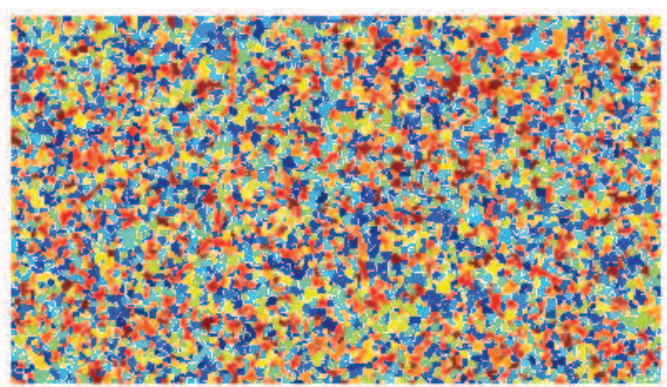

Segmentation method using gradients

Figure 3: Over-segmentations produced by the general segmentation method and gradients segmentation method 


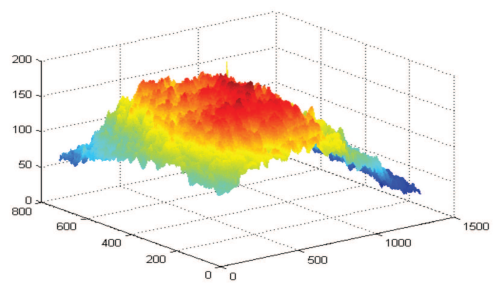

Mesh with color proportional to height

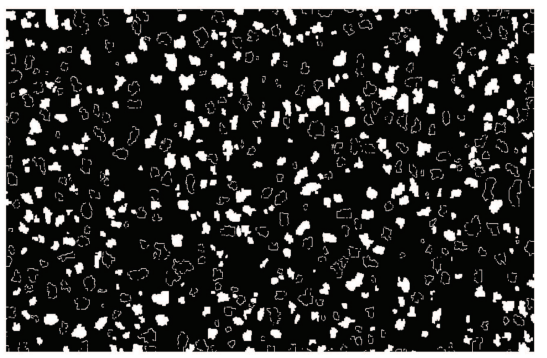

Binary image of marked matrix

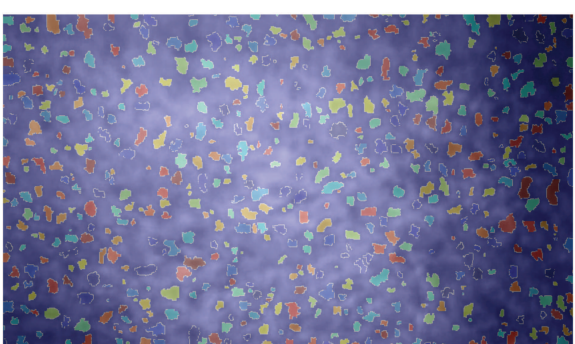

Marked matrix by the watershed transform

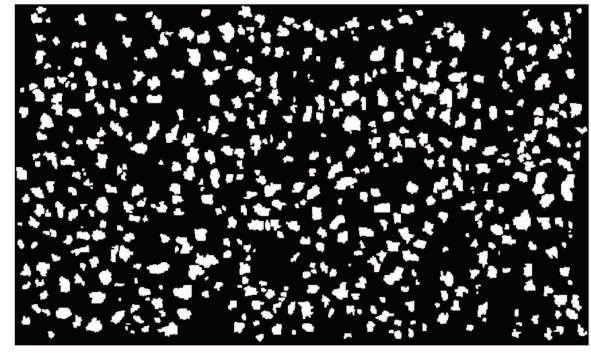

Filling with holes and clearing border

Figure 4: Marker-controlled multiple watershed segmentations combined with thresholding and filling operations 


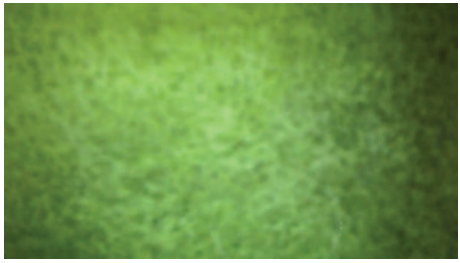

Color image

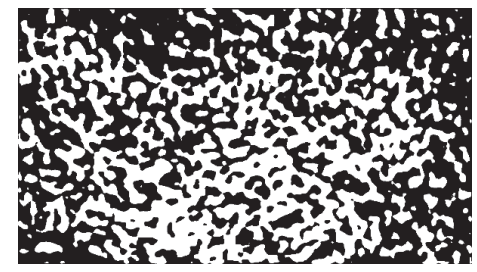

Binary image by Huang method $\beta_{0}=55, \beta_{1}=165$

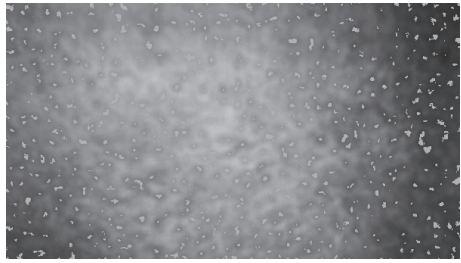

Marked region near the minimum value in gray image

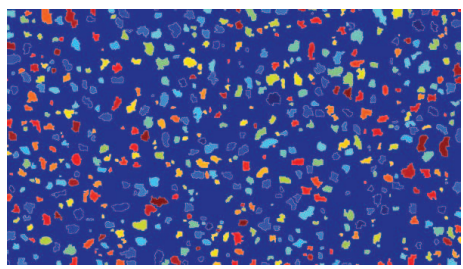

Color image marked by the watershed transform $\beta_{0}=1 \cdot \beta_{1}=529$

Figure 5: Homological characterization of gas-liquid two phase flow patterns by Huang method and watershed transform

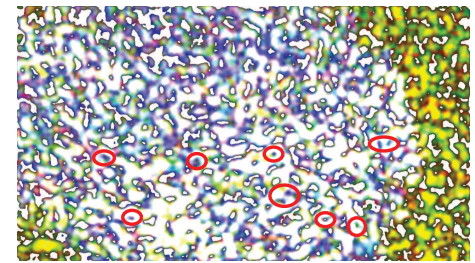

Segmentation image by SVM method

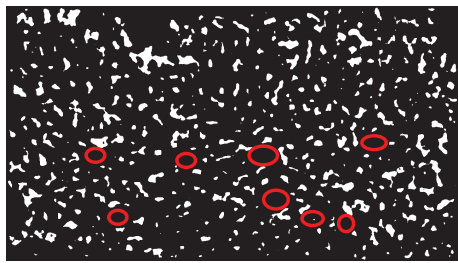

Binary image by SVM method

Figure 6: Homological characterization of gas-liquid two phase flow patterns by SVM method 


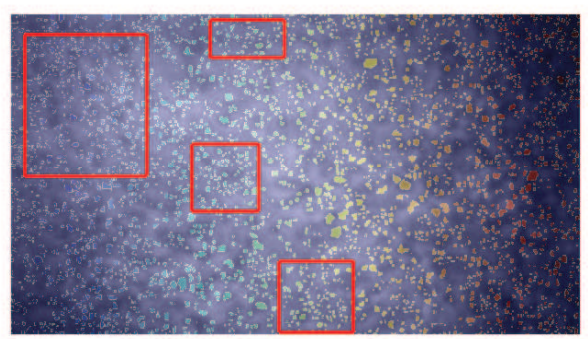

The area near the minimum value with distance $=0$

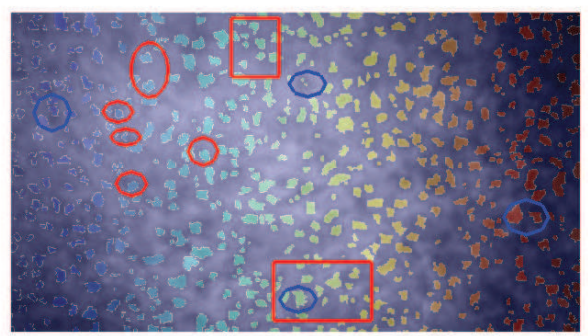

The area near the minimum value with distance $=2$

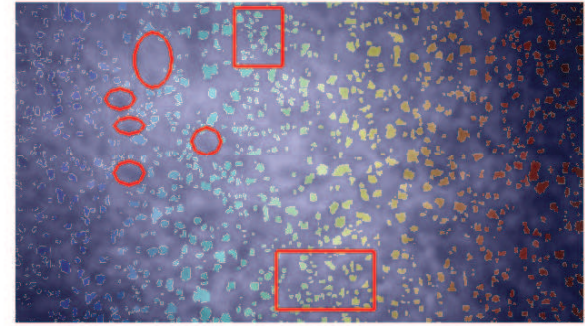

The area near the minimum value with distance $=1$

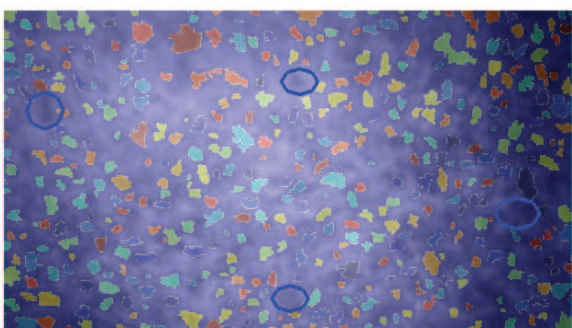

The area near the minimum value with distance $=3$

Figure 7: Influence of area near the minimum value on flow regimes identification
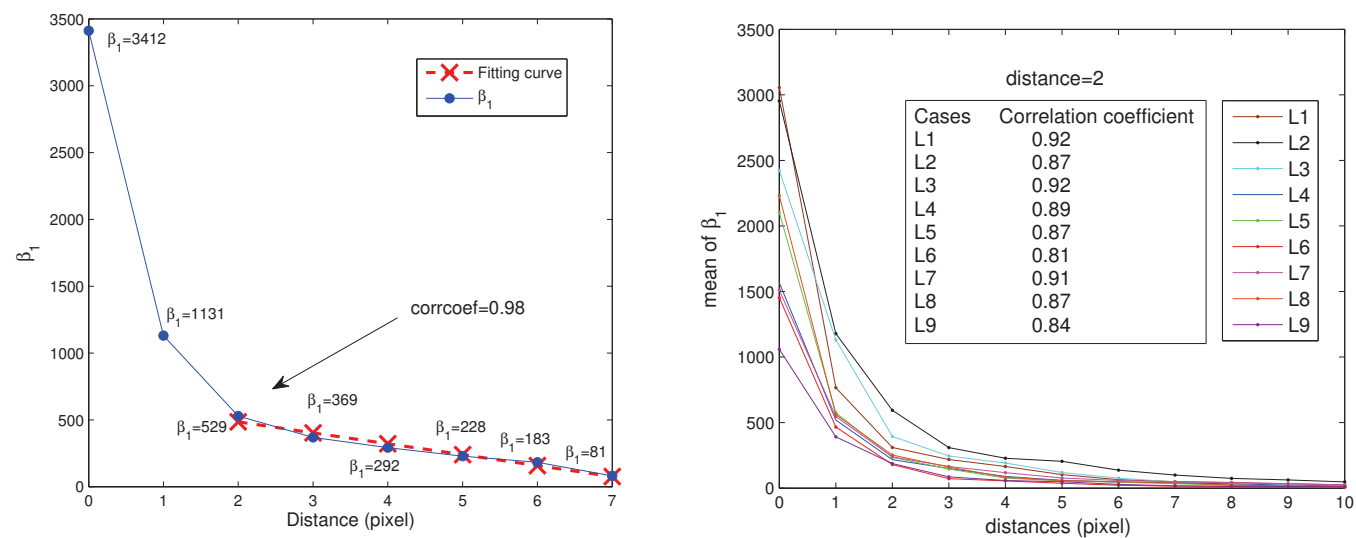

Figure 8: Relationship between distance near the minimum value and betti number of continuous phase with a good correlation coefficient (distance $=2$ ) 


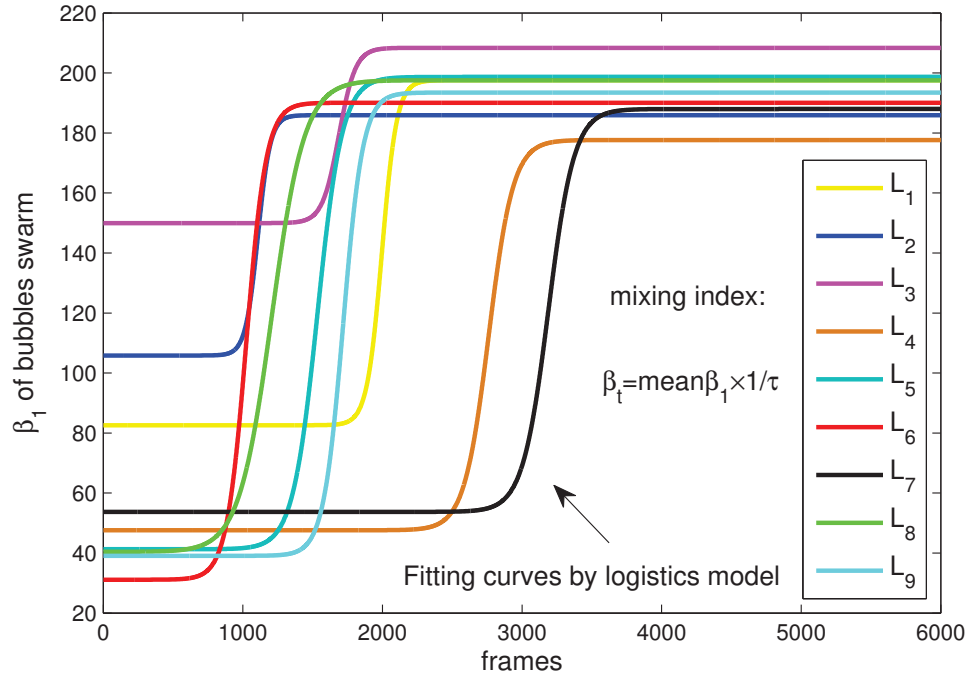

Figure 9: Plots of the bubbles swarm evolution by using a four-parameter logistics model 

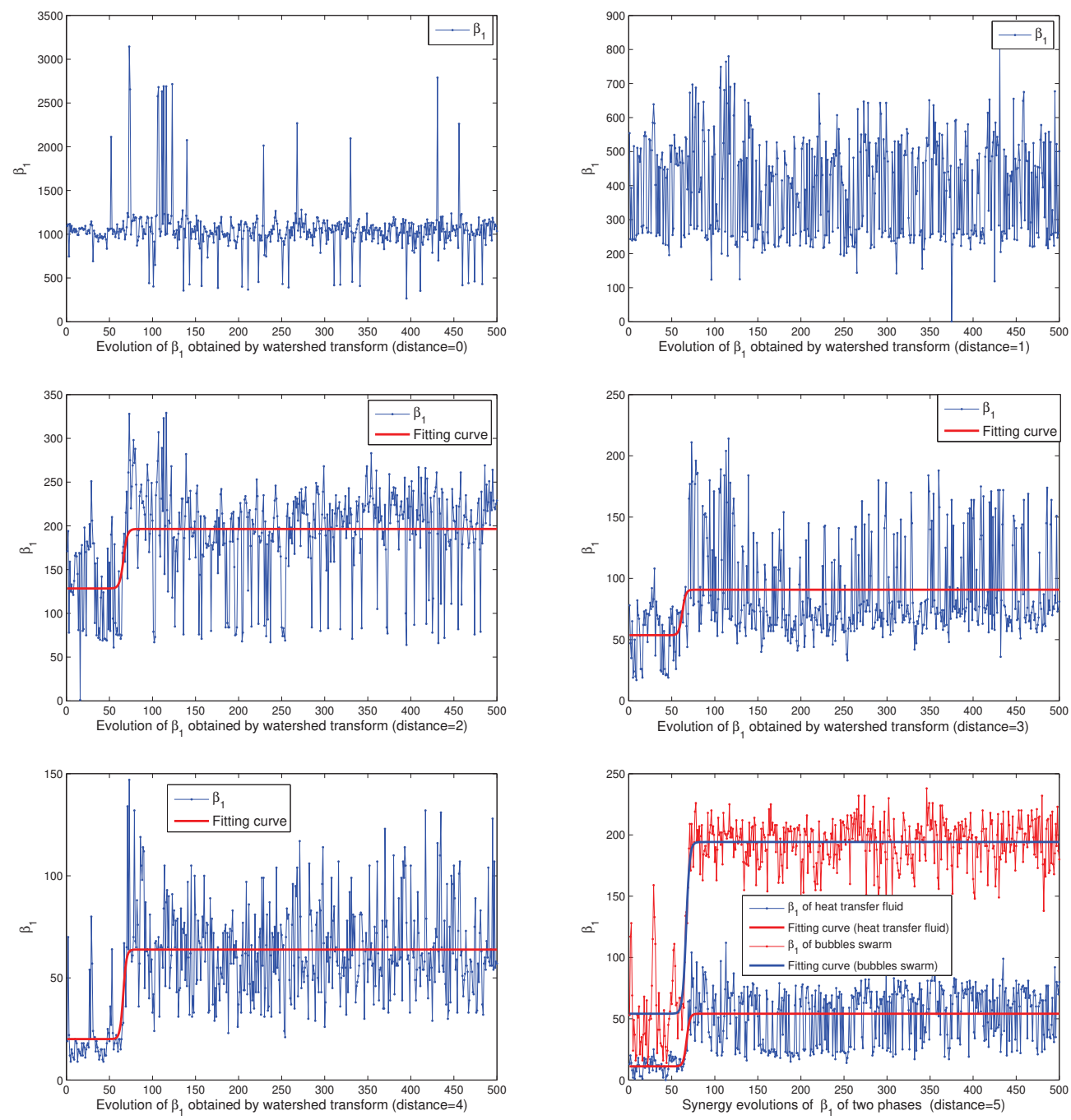

Figure 10: Evolution curves of betti number of heat transfer fluid with an increasing distance $\left(L_{9}\right)$ 

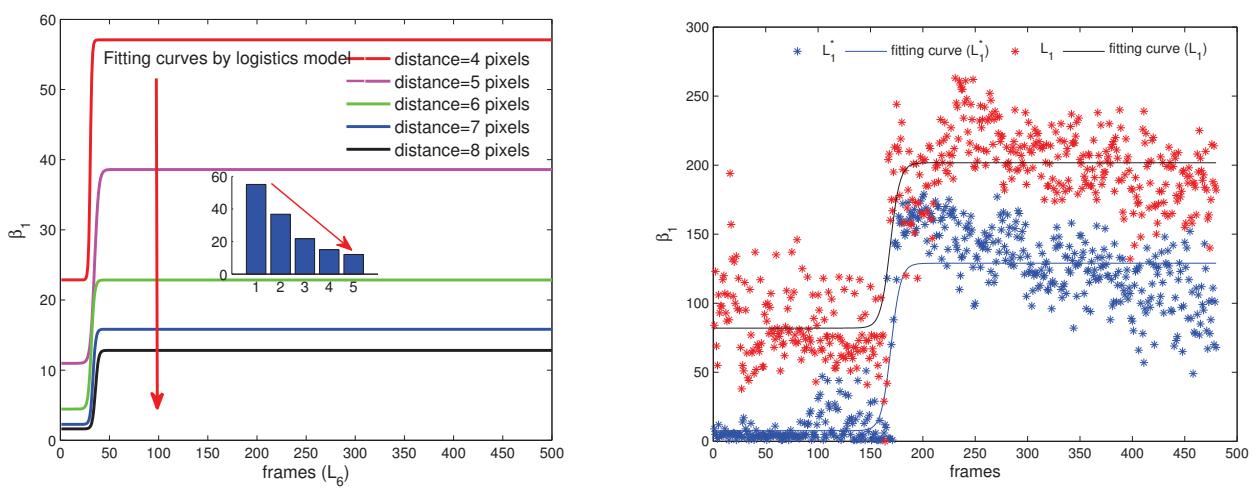

Figure 11: Influence of distance of pixels on fitting curves by logistics model (Left); Influence of repeated experiment on Logistic curve fitting (Right)
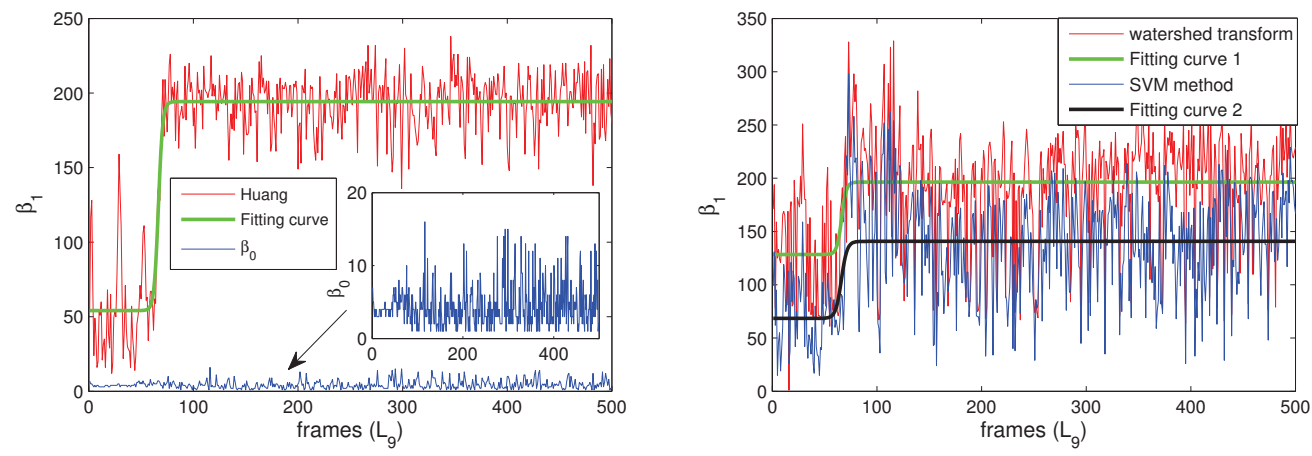

Figure 12: Evolution plots of the betti numbers of heat transfer fluid (blue) by Huang method (Left), SVM and watershed transform (Right) 

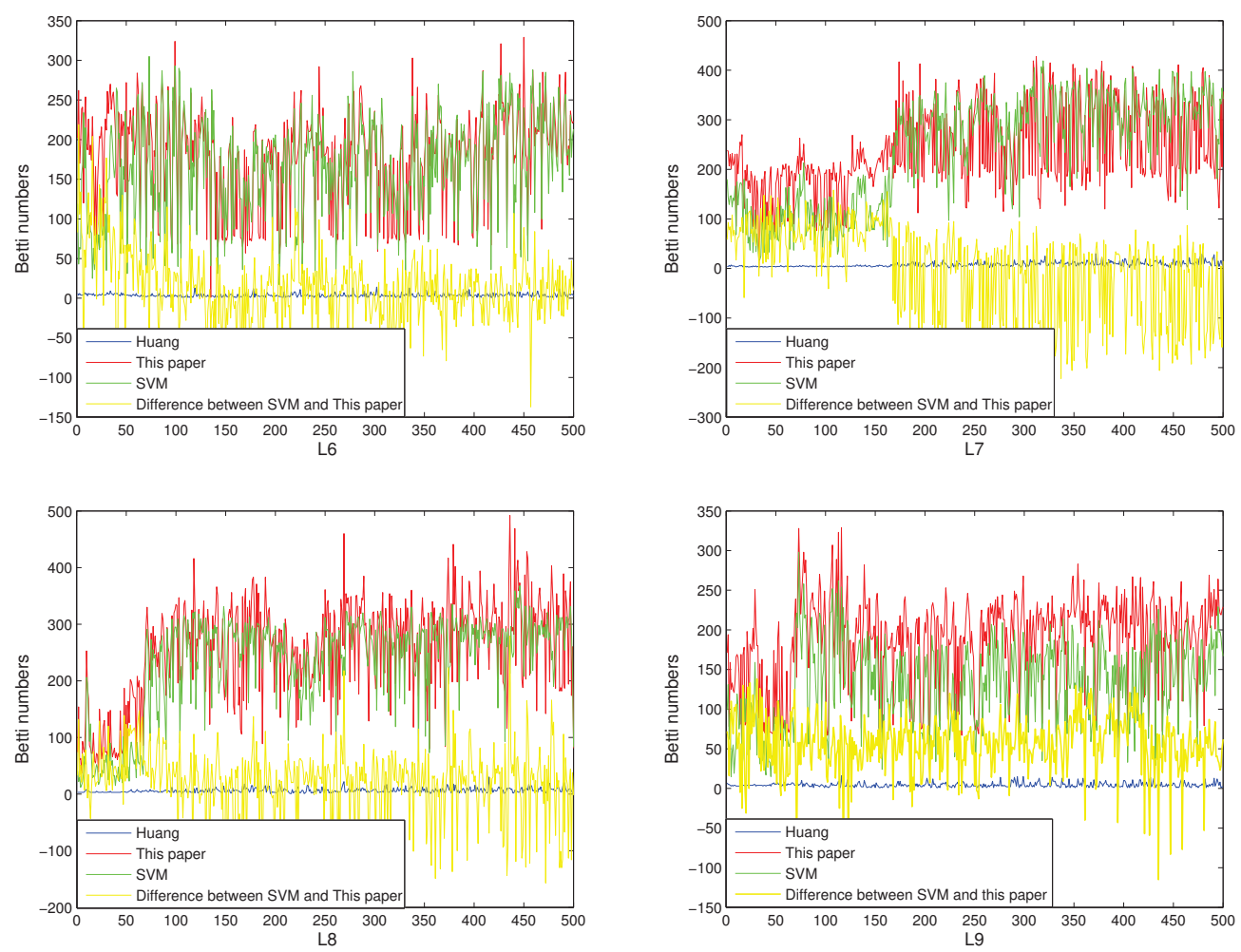

Figure 13: Evolution plots of continuous phase topological invariants by Huang method, SVM and watershed transform 

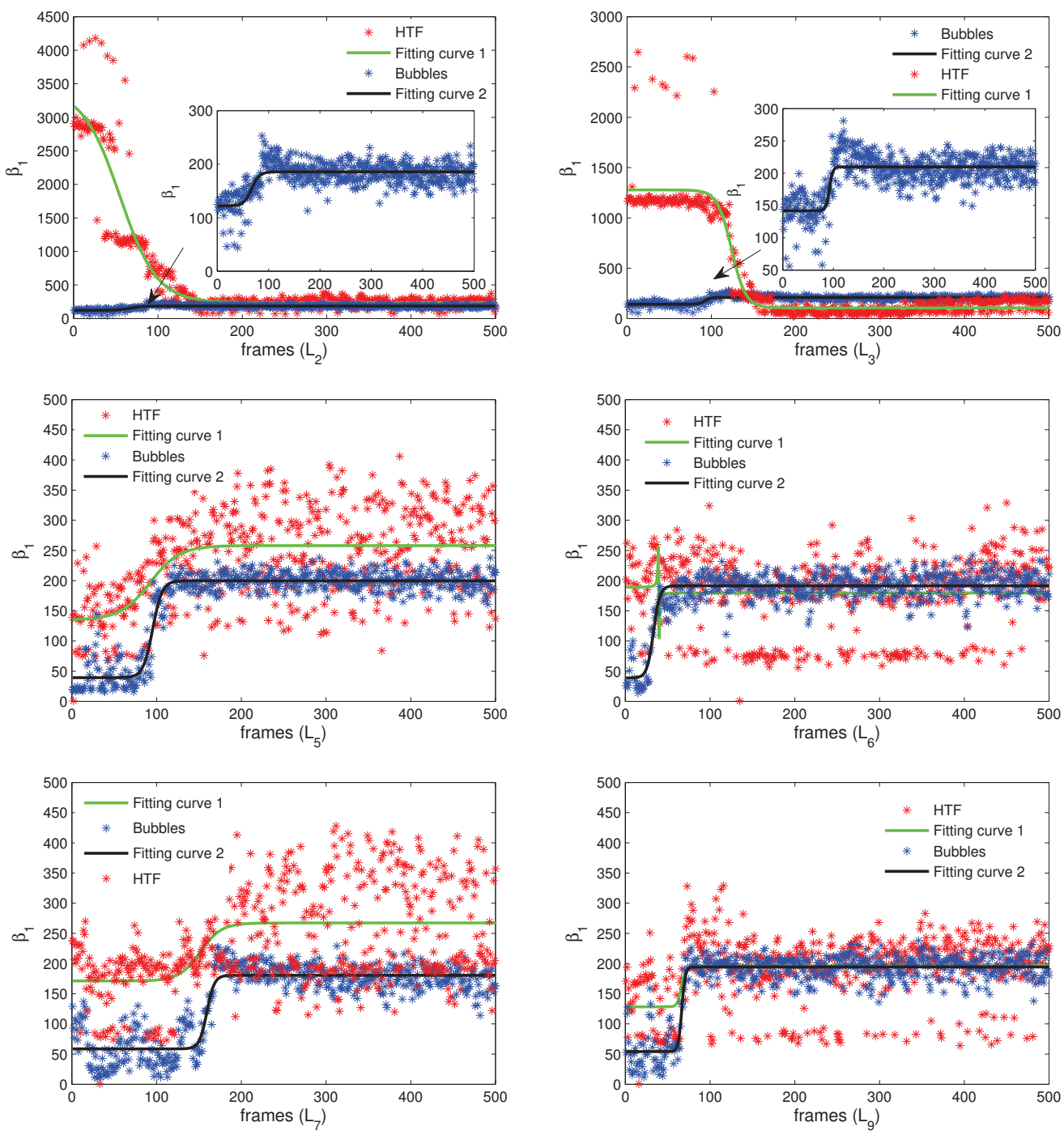

Figure 14: Topological evolution and synchronization plots of HTF phase and bubbles using four-parameter logistic regression model 

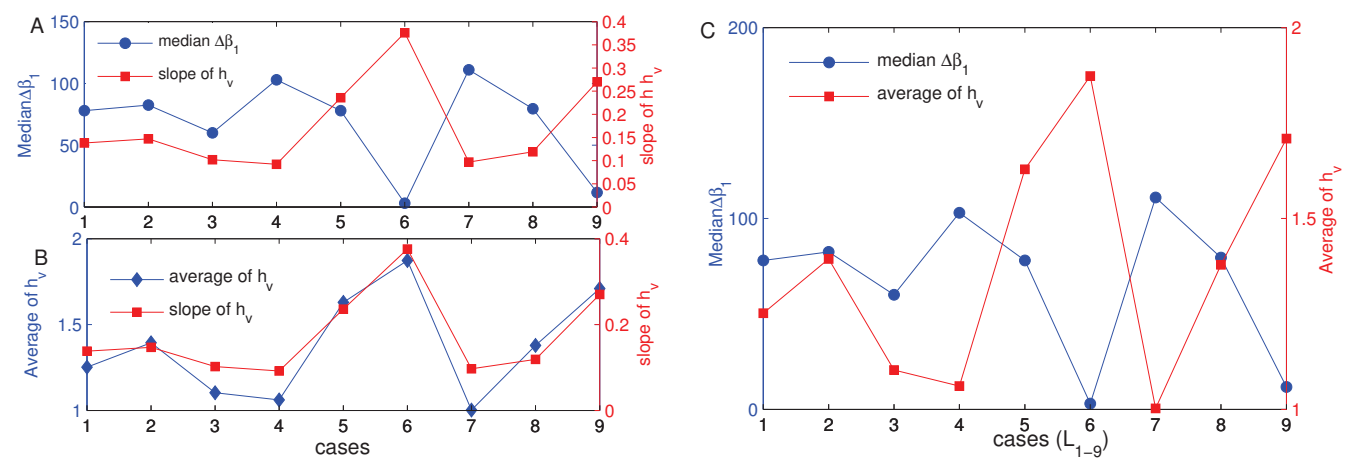

Figure 15: Topological coupling of gas-liquid two-phase flow patterns and heat exchanger efficiency. 


\begin{tabular}{llllll}
\hline Factors & Design parameters & units & level 1 & level 2 & level 3 \\
\hline A & Height of HTF $(Z)$ & $\mathrm{m}$ & 0.4 & 0.5 & 0.6 \\
B & Initial temperature difference $(\Delta T)$ & ${ }^{\circ} \mathrm{C}$ & 80 & 100 & 120 \\
C & Working fluid flow rate $\left(U_{0}\right)$ & $\mathrm{ms}^{-1}$ & 0.04 & 0.06 & 0.08 \\
$\mathrm{D}$ & $\mathrm{HTF}$ flow rate $\left(U_{c}\right)$ & $\mathrm{kgs}^{-1}$ & 0 & 0.15 & 0.3 \\
\hline
\end{tabular}

Table 1: Designing parameters and levels

\begin{tabular}{lllll}
\hline Test numbers & A & B & C & D \\
\hline$L_{1}$ & 1 & 1 & 1 & 1 \\
$L_{2}$ & 1 & 2 & 2 & 2 \\
$L_{3}$ & 1 & 3 & 3 & 3 \\
$L_{4}$ & 2 & 1 & 2 & 3 \\
$L_{5}$ & 2 & 2 & 3 & 1 \\
$L_{6}$ & 2 & 3 & 1 & 2 \\
$L_{7}$ & 3 & 1 & 3 & 2 \\
$L_{8}$ & 3 & 2 & 1 & 3 \\
$L_{9}$ & 3 & 3 & 2 & 1 \\
\hline
\end{tabular}

Table 2: $\left(L_{9}\left(3^{4}\right)\right.$ orthogonal arrays

\begin{tabular}{llllllllll}
\hline Index & $L_{1}$ & $L_{2}$ & $L_{3}$ & $L_{4}$ & $L_{5}$ & $L_{6}$ & $L_{7}$ & $L_{8}$ & $L_{9}$ \\
\hline slopeof $h_{v}$ & 0.138 & 0.147 & 0.102 & 0.092 & 0.236 & 0.376 & 0.097 & 0.119 & 0.27 \\
median $\left(\Delta \beta_{1}\right)$ & 78 & 82.5 & 60 & 103 & 78 & 3 & 111 & 79.5 & 11.75 \\
$\overline{h_{v}}$ & 1.251 & 1.393 & 1.103 & 1.061 & 1.628 & 1.873 & 1.002 & 1.378 & 1.709 \\
\hline
\end{tabular}

Table 3: Growth rate of heat exchanger efficiency, the median of $\Delta \beta_{1}$ of gas-liquid twophase flow patterns and the average of heat exchanger efficiency. 